\title{
Magnetic Gelatin Microspheres for Targeted Release of Doxorubicin
}

\author{
Josefa Virginia da Silva Souza ${ }^{a}$, Manoel Ribeiro da Silva ${ }^{b}$, Marcos Antonio da Silva Costa, ${ }^{a, *}$ \\ ${ }^{a}$ Universidade do Estado do Rio de Janeiro, Programa de Pós-graduação em Química, Instituto de \\ Química, 20550-900, Rio de Janeiro, RJ, Brasil. \\ ${ }^{b}$ Universidade Federal de Itajubá, Instituto de Física e Química, 37500-903, Itajubá, MG, Brasil.
}

Received: April 12, 2021; Revised: July 15, 2021; Accepted: September 04, 2021

\begin{abstract}
The aims of this work were to develop a magnetic carrier system consisting of microspheres made of gelatin for controlled release of doxorubicin and to investigate the ability of fructose, glucose, genipin and 1-ethyl-3(3-dimethylaminopropyl)carbodiimide (EDC) as crosslinking agents of gelatin microspheres. In addition, we also investigated the changes in the microspheres' magnetic properties produced by using magnetite incorporated by three different methods and the doxorubicin release performance by using a constant, oscillating or no external magnetic field. The system obtained with fructose as crosslinker (low toxicity) was superparamagnetic, with high saturation magnetization and release profiles that could be controlled. Among the crosslinkers studied, genipin was the most efficient to reduce the solubility of the gelatin microspheres in water. When ferrous chloride was employed in magnetite synthesis, the Ms showed the highest values $(61.6 \mathrm{emu} / \mathrm{g})$, and its use in magnetic gelatin microspheres allowed the production of systems with high magnetization $(29.6 \mathrm{emu} / \mathrm{g})$.
\end{abstract}

Keywords: Magnetic, Gelatin, Microspheres, Release, Doxorubicins.

\section{Introduction}

Targeted and controlled drug delivery systems have been studied by many researchers in the areas of medicine, pharmaceuticals and chemistry ${ }^{1-3}$. The reason for this research interest is their advantages over conventional chemotherapeutic drugs, especially anti-cancer drugs. When particles (nano and microspheres, fibers or implants) loaded with anti-cancer drugs are delivered to a targeted area, the undesired side-effects are reduced and the drug concentration in target tissues can be increased while minimizing toxicity, enhancing drug efficacy ${ }^{4-6}$. Polymeric materials have long been used to produce these particles, especially microspheres for biomedical applications, by virtue of their small size and efficient carrier characteristics ${ }^{7}$. One of the most popular polymeric materials for this purpose is gelatin. It offers a number of benefits: biocompatibility, biodegradability, low antigenicity, low cost, numerous available active groups for attaching targeting molecules and ease of use in parenteral formulations ${ }^{8-12}$. However, because it is water soluble, gelatin has to be modified to prepare drug delivery systems. This modification involves a crosslink reaction to connect the protein chains with each other and decrease their water solubility. For this purpose, glutaraldehyde (GTA) has often been studied as crosslinking agent ${ }^{13-16}$. GTA use is attractive because it is easily available, inexpensive and can effectively crosslink collagenous tissues rapidly ${ }^{17}$. However, the biodegradation of gelatin crosslinked with GTA particles can cause GTA release inside the body and undesired effects because of its cytotoxicity $^{18,19}$. Different crosslinking agents have been reported for gelatin to alter the toxicity associated with

*e-mail: marcoscosta.iq.uerj@gmail.com crosslinking agents ${ }^{19-25}$. Here we evaluate fructose, glucose, genipin and 1-ethyl-3(3-dimethylaminopropyl)carbodiimide (EDC) as crosslinking agents in gelatin microspheres because of their low cytotoxicity.

Another property that should be observed in controlled drug delivery systems is how the drug is transported to the target cells. The particles containing the drug should come in contact with the diseased tissue as quickly as possible and follow the shortest route to keep the drug from being released in undesired places. Widder et al. ${ }^{26}$ first described the use of magnetically responsive biodegradable drug carriers with the ability to direct both carrier and therapeutic agents by magnetic means to a specified target site. Since then, many other papers have studied the use of magnetic materials in targeted drug delivery systems. In general, magnetic microspheres are infused into an artery supplying a given target site. A magnet with sufficient field strength to retard the microspheres at the capillary level is placed externally over the target area ${ }^{27}$. Because of arterial administration with subsequent magnetic targeting, the majority of infused microspheres do not circulate systemically and can be retained to achieve very high concentrations of the chemotherapeutic agent near the target site for a given period of time without any toxic effects to normal surrounding tissue or the whole body ${ }^{4,5,16}$. The magnetic material most widely used for this aplication is magnetite because of its high levels of saturation magnetization and biocompatibility ${ }^{28}$. For this reasons, magnetite was used in the gelatin microspheres produced in this study.

Doxorubicin is an anthracycline cytostatic antibiotic with activity against a variety of malignancies, especially in the treatment of soft tissue and bone sarcoma ${ }^{29}$. Like other 
cancer drugs, doxorubicin is very toxic and has a narrow therapeutic index ${ }^{30}$. Thus, it was evaluated in this work.

The presence of multifunctional groups, like $-\mathrm{NH}_{2}$ and $-\mathrm{COOH}$, in the gelatin chain makes magnetite a suitable candidate to bind with drugs like doxorubicin (DXR), forming a drug-polymer conjugate ${ }^{8}$. Although application of magnetite for doxorubicin targeted delivery systems based on gelatin has already been investigated by many researchers, its comparison with fructose, glucose, genipin and EDC to establish which is the best low cytotoxic crosslinker has not been reported to date. There have also been no reports of the influence of different external magnetic fields on the kinetics of doxorubicin release. Thus, the aims of this work were to develop a magnetic carrier system composed of microspheres made of gelatin for controlled release of doxorubicin and to investigate the ability of fructose, glucose, genipin and EDC to crosslink gelatin microspheres.

\section{Materials and Methods}

\subsection{Materials}

Type B gelatin (225 bloom), fructose, glucose, corn oil, 1-ethyl-3-(3-dimethylaminopropyl) carbodiimide (EDC) and doxorubicin were purchased from Sigma-Aldrich Co. Genipin was purchased from Cayman. Acetone, potassium dihydrogen phosphate, sodium hydrogen phosphate, sodium chloride, copper sulphate, sodium and potassium double tartrate and sodium hydroxide were acquired from Vetec Química Fina Ltda. Ferric chloride, sodium chloride and ferrous sulfate were purchased from Proquimios Comércio e Indústria Ltda. All chemicals were analytical grade and used as received.

\subsection{Preparation of gelatin microspheres crosslinked with fructose or glucose}

The gelatin microspheres were produced by thermal gelation. Typically, $1 \mathrm{~g}$ of gelatin, $10 \mathrm{~mL}$ of distilled water and $1 \mathrm{~g}$ or $0,5 \mathrm{~g}$ (according to design of experiments) of sugar were added to a $100 \mathrm{~mL}$ beaker. This mixture was preheated to $80{ }^{\circ} \mathrm{C}$ for 10 minutes. Then, it was added dropwise to a $500 \mathrm{~mL}$ threenecked round-bottom flask equipped with a mechanical stirrer $(500 \mathrm{rpm})$ containing $40 \mathrm{~mL}$ of corn oil to form an emulsion. When the emulsion was obtained, the temperature was kept at $80^{\circ} \mathrm{C}$ for different time periods ( 10 or 30 minutes) and then quickly lowered to $5^{\circ} \mathrm{C}$ by immersion in an ice bath for 30 minutes. Then, to completely solidify the droplets of the dispersed phase, $50 \mathrm{~mL}$ of precooled $\left(5^{\circ} \mathrm{C}\right)$ acetone was added and the mixture was stirred for another hour. The microspheres were filtered, washed with cool acetone $\left(5^{\circ} \mathrm{C}\right)$ and rapidly dried.

\subsection{Preparation of gelatin microspheres crosslinked with genipin, glutaraldehyde or EDC}

Microspheres were produced in the same way as described in 2.2, but without crosslinkers. The non-crosslinked gelatin microspheres were put in contact with a solution of genipin, EDC or glutaraldehyde, in all cases at a concentration of $3.6 \mathrm{mM}$, for different time periods at $37^{\circ} \mathrm{C}$.

\subsection{Preparation of magnetite}

\subsubsection{Magnetite obtained from ferrous sulfate}

Magnetite nanoparticles were synthesized using an adaptation of a previously described co-precipitation method ${ }^{31}$. In a becker, $64 \mathrm{~mL}$ of $\mathrm{FeCl}_{3}(0.5 \mathrm{M})$ aqueous solution and $36 \mathrm{~mL}$ of $\mathrm{a} \mathrm{FeSO}_{4}(0.5 \mathrm{M})$ aqueous solution were mixed under mechanical stirring (400 rpm) and nitrogen atmosphere. Then, $10 \mathrm{~mL}$ of an aqueous ammonia solution (25\%) were then quickly poured into the solution, followed by more $50 \mathrm{~mL}$ of ammonia solution, this time dripped slowly into the mixture. The solution was stirred for an additional $30 \mathrm{~min}$ under heating at $90{ }^{\circ} \mathrm{C}$. The dispersion was cooled to room temperature and washed several times with distilled water until neutral $\mathrm{pH}$. The magnetite formed was separated by magnetic decantation/separation and lyophilized for $24 \mathrm{~h}$. The $\mathrm{Fe}_{3} \mathrm{O}_{4}$ nanoparticles' precipitation happened according to the equation 1 :

$$
\mathrm{Fe}^{2+}+2 \mathrm{Fe}^{3+}+8 \mathrm{OH}^{-} \rightarrow \mathrm{Fe}_{3} \mathrm{O}_{4}+4 \mathrm{H}_{2} \mathrm{O} \quad \text { Equation } 1
$$

\subsubsection{Magnetite obtained from ferrous chloride}

The magnetite nanoparticles were produced in the same way described in 2.4.1, but utilizing a $\mathrm{FeCl}_{2}$ instead of $\mathrm{FeSO}_{4}$. Thus, $64 \mathrm{~mL}$ of $\mathrm{FeCl}_{3}(0.5 \mathrm{M})$ aqueous solution and $36 \mathrm{~mL}$ of a $\mathrm{FeCl}_{2}(0.5 \mathrm{M})$ aqueous solution were mixed under mechanical stirring $(400 \mathrm{rpm})$ and nitrogen atmosphere. Then, $10 \mathrm{~mL}$ of an aqueous ammonia solution (25\%) were then quickly poured into the solution, followed by more $50 \mathrm{~mL}$ of ammonia solution, this time dripped slowly into the mixture. The solution was stirred for an additional $30 \mathrm{~min}$ under heating at $90{ }^{\circ} \mathrm{C}$. The dispersion was cooled to room temperature and washed several times with distilled water until neutral $\mathrm{pH}$.

\subsubsection{Magnetic fluid based on magnetite obtained from ferrous sulfate}

The preparation of magnetic fluid was performed as the following: $95.5 \mathrm{~mL}$ of $\mathrm{FeCl}_{3}(0.5 \mathrm{M})$ aqueous solution and $54.5 \mathrm{~mL}$ of $\mathrm{FeSO}_{4}(0.5 \mathrm{M})$ aqueous solution were mixed under mechanical stirring $(400 \mathrm{rpm})$ and nitrogen atmosphere. The mixed solution was heated to $50{ }^{\circ} \mathrm{C}$ and stirred at $300 \mathrm{rpm}$. After that, $7.5 \mathrm{~g}$ of gelatin were added. After complete gelatin solubilization, $10 \mathrm{~mL}$ of an aqueous ammonia solution $(25 \% \mathrm{w} / \mathrm{v})$ were then quickly poured into the solution under vigorous stirring, followed by $50 \mathrm{~mL}$ more of the solution, dripped slowly into the mixture with mechanical stirring (400 rpm). Then the solution was stirred for an additional $30 \mathrm{~min}$ under heating $\left(50^{\circ} \mathrm{C}\right)$. The fluid was cooled to room temperature and stored for later use.

\subsection{Preparation of the magnetic gelatin microspheres}

Magnetic microspheres were produced by the same method described in 2.2. Typically, $1 \mathrm{~g}$ of gelatin, $10 \mathrm{~mL}$ of distilled water, $1 \mathrm{~g}$ of fructose and $1 \mathrm{~g}$ of magnetic material (magnetite or magnetic fluid) were added to a $100 \mathrm{~mL}$ beaker. This mixture was preheated to $80^{\circ} \mathrm{C}$ for 10 minutes. Then, it was added dropwise to a $500 \mathrm{~mL}$ threenecked round-bottom 
flask equipped with a mechanical stirrer ( $500 \mathrm{rpm})$ containing. $40 \mathrm{~mL}$ of corn oil to form an emulsion. When the emulsion was obtained, the temperature was kept at $80^{\circ} \mathrm{C}$ for different time periods (10 or 30 minutes) and then quickly lowered to $5{ }^{\circ} \mathrm{C}$ by immersion in an ice bath for 30 minutes. Then, to completely solidify the droplets of the dispersed phase, $50 \mathrm{~mL}$ of precooled $\left(5^{\circ} \mathrm{C}\right)$ acetone was added and the mixture was stirred for another hour. The microspheres were filtered, washed with cool acetone $\left(5^{\circ} \mathrm{C}\right)$. Then, drying was carried out in a desiccator at room temperature.

\subsection{Magnetic properties}

The magnetic properties (saturation magnetization $(M s)$, coercive field $(\mathrm{Hc})$ and and hysteresis loop) were measured at room temperature by using a Lake Shore series 7400 vibrating sample magnetometer (VSM). The hysteresis loops were measured under a magnetic field strength of 10,000 Gauss at room temperature. The data were taken with 80 points/ loop with a scan speed $10 \mathrm{~s} /$ point.

\subsection{Water solubility tests}

Biuret reagent was prepared by $1.5 \mathrm{~g}$ of copper sulphate $\left(\mathrm{CuSO}_{4} .5 \mathrm{H}_{2} \mathrm{O}\right)$ and $6.0 \mathrm{~g}$ of sodium and potassium double tartrate $\left(\mathrm{KNaC}_{4} \mathrm{H}_{4} \mathrm{O}_{6} \cdot 4 \mathrm{H}_{2} \mathrm{O}\right)$ dissolution in $500 \mathrm{ml}$ of distilled water. Then, $300 \mathrm{~mL}$ of $10 \% \mathrm{NaOH}$ solution and $1 \mathrm{~g}$ of potassium iodide (KI) were added under magnetic stirring. The volume was completed to $1 \mathrm{~L}$ with distilled water.

In order to perform water solubility tests, about $0.4 \mathrm{~g}$ of gelatin microspheres were transferred to a $50-\mathrm{mL}$ roundbottom flask. The volume in the beaker was completed with purified water (Milli-Q) and the beaker was then placed in a water bath at $37^{\circ} \mathrm{C}$ for one hour. After heating, the sample was cooled to room temperature and filtered through a polyethersulfone membrane pore size of $0.45 \mu \mathrm{m}$. The filtrate was reserved for subsequent analysis of concentration of proteins. This procedure was repeated for all the samples. A $0.2-\mathrm{mL}$ aliquot of the filtrate obtained in the dissolution test was placed in a test tube to react with $5.0 \mathrm{~mL}$ of Biuret reagent for 30 minutes in the dark. Then the absorbances of the solutions obtained were read with a Fentom 600S spectrophotometer at $550 \mathrm{~nm}$. Each sample was tested in triplicate.

\subsection{Enzymatic degradation}

In a crucible, about $100 \mathrm{mg}$ of gelatin microspheres were placed in contact with a phosphate buffer saline (PBS) (pH 7.4, $1.2 \mathrm{mM} \mathrm{KH}_{2} \mathrm{PO}_{4}, 1.15 \mathrm{mM} \mathrm{Na} \mathrm{HPO}_{4}, 2.7 \mathrm{mM}$ $\mathrm{KCl}, 1.38 \mathrm{mM} \mathrm{NaCl}$ ) containing $30 \mathrm{mg} / \mathrm{mL}$ of enzyme of collagenase at $37{ }^{\circ} \mathrm{C}$ for 24 hours. After this period, the samples were allowed to cool to room temperature. Then the gelatine microspheres were separated by filtration and dried in a desiccator for 24 hours. Finally, the samples were weighed and the percentage of weight loss was calculated. Each sample was tested in triplicate.

\subsection{Morphology of the microspheres}

The morphology of the gelatin microspheres was investigated with a Carl Zeiss Leo 1450 VPO scanning electron microscope (SEM). The samples were coated with a thin layer of gold to increase their conductivity and protect them against localized heating. The images were captured using secondary electron detector with work distance of $9 \mathrm{~mm}$, spot size of $20 \mathrm{~nm}$ and electron acceleration voltage of $5 \mathrm{kV}$. The samples were mounted on an aluminum stub with carbon adhesive, coated with $40 \mathrm{~nm}$ of gold and then observed in the microscope.

\subsection{Drug loading}

We used an adaptation of a previously described method ${ }^{32}$. About $300 \mathrm{mg}$ of microspheres were placed in a doxorubicin solution $(0.5 \mathrm{mg} / \mathrm{mL})$ for different time periods. After the predetermined time, the absorbance of each doxorubicin solution was measured with a Fentom 600 S spectrophotometer at $496 \mathrm{~nm}$. The amount of doxorubicin stored by the microspheres was calculated using the Equation 2:

$C=C o-C f$

Where $\mathrm{C}_{0}$ is the initial concentration of doxorubicin and $\mathrm{C}_{\mathrm{f}}$ is the final concentration, both obtained by calibration curve. Calibration samples were prepared fresh by serial dilution of doxorubicin standard solution $(0.5 \mathrm{mg} / \mathrm{mL})$. The final drug concentration range was $0.01-0.5 \mathrm{mg} / \mathrm{mL}$. Samples were prepared independently in triplicate.

\subsection{In vitro release experiment}

Release experiments were performed in triplicate using the method described by Choubey and Bajpai ${ }^{33}$ in phosphate buffer saline (PBS) in the absence or presence of a magnetic field (constant or oscillatory). To determine the amount of doxorubicin (DOX) released, $8 \mathrm{~mL}$ of PBS were added to $0.1 \mathrm{~g}$ of drug-loaded nanoparticles as a release medium ( $\mathrm{pH}$ 7.4) and the resulting suspension was gently shaken for a predetermined time period. After shaking, $3 \mathrm{~mL}$ of supernatant were withdrawn and assayed for DOX spectrophotometrically.

\section{Results and Discussion}

Gelatin systems dissolve relatively quickly when placed in aqueous environments. Because of this, the crosslinking of gelatin chains is one of the most important experimental parameters that has been analysed to produce drug release systems. In this study, one of the goals was to replace traditional crosslinking agents (glutaraldehyde, glyceraldehyde and other petroleum products) with agents having lower cytotoxicity. Therefore, we evaluated the crosslinking action of fructose, glucose, EDC and genipin and comparing them with glutaraldehyde.

\subsection{Gelatin crosslink using fructose and glucose}

Preliminary tests showed that due to the cyclization that occurs with saccharides, their reaction kinetics is very slow, so some experimental factors need to be changed in order to maximize the crosslinking reaction efficiency when using fructose or glucose. For this purpose, we performed fractional factorial experiments of the $2^{4,1}$ type with two central points and duplicate at these central points. The dissolution profile in aqueous media at $37^{\circ} \mathrm{C}$ was chosen as the dependent variable because crosslinking degree of the sample and its dissolution percentage are inversely proportional. Table 1 shows the 
Table 1. Composition and dissolved percentage of samples.

\begin{tabular}{cccccc}
\hline Sample & Crosslinker & Heating time (min) & $\begin{array}{c}\text { Crosslinker / gelatine } \\
\text { (weight ratio) }\end{array}$ & $\mathrm{pH}$ & Dissolved \% \\
\hline 1 & glucose & 30 & $50: 100$ & 8 & 70.11 \\
\hline 2 & fructose & 10 & $50: 100$ & 8 & 60.19 \\
\hline 3 & glucose & 10 & $50: 100$ & 9 & 80.83 \\
\hline 4 & glucose & 10 & $100: 100$ & 8 & 50.5 \\
\hline 5 & fructose & 30 & $50: 100$ & 9 & 76.30 \\
\hline 6 & glucose & 30 & $100: 100$ & 9 & 73.45 \\
\hline 7 & fructose & 10 & $100: 100$ & 9 & 57.01 \\
\hline 8 & fructose & 30 & $100: 100$ & 8 & 26.85 \\
\hline central 1 & glucose & 20 & $75: 100$ & 8.5 & 60.26 \\
\hline central 1 & glucose & 20 & $75: 100$ & 8.5 & 81.92 \\
\hline central 2 & fructose & 20 & $75: 100$ & 8.5 & 73.58 \\
\hline
\end{tabular}

Table 2. Analysis of variance for a $2^{4,1}$ factorial experimental design.

\begin{tabular}{ccccc}
\hline & Sum of squares & Mean square & $\mathrm{F}^{1}$ & $\mathrm{p}^{\text {-value }}{ }^{2}$ \\
\hline Crosslinker & 214.377 & 2.143 .765 & 1.747 .292 & 0.227776 \\
\hline Time & 0.410 & 0.4095 & 0.003338 & 0.955544 \\
\hline Crosslinker concentration( \%) & 792.219 & 7.922 .190 & 6.457 .040 & 0.038604 \\
\hline pH & 798.601 & 7.986 .006 & 6.509 .054 & 0.038032 \\
\hline Error & 858.835 & 1.226 .907 & & \\
\hline Total SS & 2.664 .441 & & & \\
\hline
\end{tabular}

$1=$ variance analysis; $2=$ significance of variance analysis.

percentage of the sample dissolved in water for each point in the planning.

Table 2 shows the analysis of variance results of the experiments. The dependent variables $\mathrm{pH}$ and crosslink percentage presented $\mathrm{p}$-values lower than the confidence limit $(\mathrm{p}<0.05)$, suggesting that these two factors are significantly affected by the samples' solubility. The response surface (Figure 1) demonstrates that sample solubility decreases with the increase in crosslinker concentration and lower $\mathrm{pH}$. The increase of crosslinker concentration caused a shift in the balance of the open chain/cyclical chain of sugars, increasing the availability of open form in the reaction medium, promoting crosslinking reactions and decreasing solubility. On the other hand, the elevation of $\mathrm{pH}$ caused increased solubility because gelatin protein chains begin to degrade at $\mathrm{pH} 9$.

Based on analysis of variance and surface response, samples with lower solubility were those produced at $\mathrm{pH} 8$ and with weight ratio crosslinker to gelatine 100:100. Samples 4 and 8 were manufactured using these experimental parameters and differed only by the type of sugar used in preparation. As can be seen in Table 1, the sample prepared with glucose (Sample 4) showed twice the solubility compared to the sample prepared with fructose (Sample 8). For this reason, we used fructose for further analysis and comparisons with other crosslinkers.

\subsection{Gelatin crosslinking using fructose, genipin and $E D C$}

Gelatin microspheres crosslinked with fructose, genipin and EDC were compared to microspheres crosslinked with

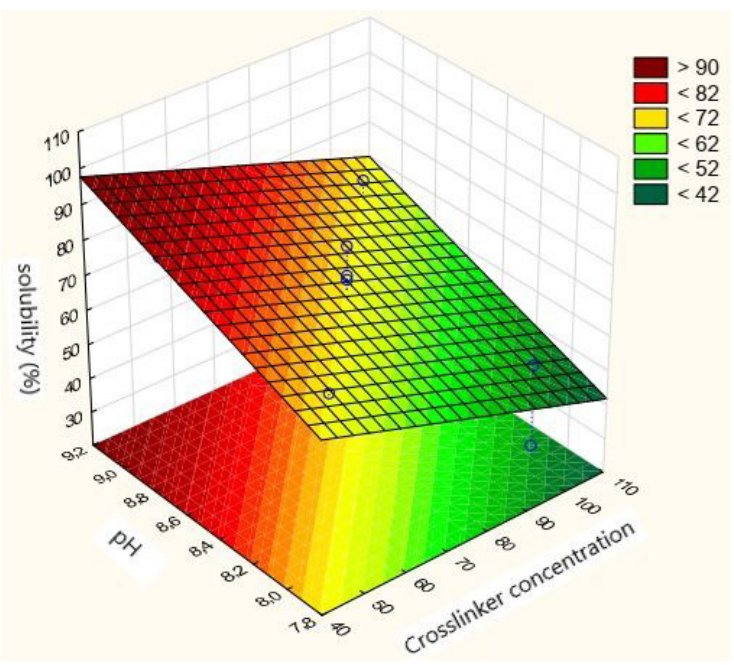

Figure 1. Response surface for dissolved percentage of samples prepared with variation in crosslinker concentration and $\mathrm{pH}$.

glutaraldehyde in order to obtain systems with lower cytotoxicity but good crosslinking degree. The samples were subjected to enzymatic degradation, water solubility and infrared analysis. The samples crosslinked with glutaraldehyde, genipin and EDC were subjected to different crosslinking times for comparative purposes. The fructose crosslinked samples were subjected only to 1 hour of crosslinking because of the high temperature and basic $\mathrm{pH}$ (necessary experimental conditions for crosslinking with sugar) cause chemical degradation of the gelatin if the exposure time is lengthy. 
The enzymatic degradation results are shown in Figure 2. More crosslinked samples are harder to degrade because the crosslinks between chains make the material stronger. The results of this analysis show that in general samples crosslinked with genipin had the lowest percentage of degradation, indicating these were more crosslinked than the others, even more than those with glutaraldehyde. The other samples crosslinked with EDC, when compared to those crosslinked with glutaraldehyde, were not significantly different after most of the intervals studied. Regarding the use of fructose as crosslinker, despite the shorter crosslinking time, its percentage of degradation (mass loss) was similar to that of glutaraldehyde after three hours. We also observed that even when compared with the other samples crosslinked with glutaraldehyde for longer than three hours, the mass loss percentage of the microspheres crosslinked with fructose was lower.

Figure 3 shows the result of the water solubility test of gelatin microspheres cross-linked with genipin, EDC, fructose or glutaraldehyde with a cross-linking reaction time of $1 \mathrm{~h}$. The samples were placed in an aqueous medium at $37^{\circ} \mathrm{C}$ and the percentage of gelatin dissolved in the medium was analyzed after $1 \mathrm{~h}, 3 \mathrm{~h}, 12 \mathrm{~h}$ and $48 \mathrm{~h}$ of immersion of the sample.

As can be seen, an increase in the percentage of the dissolution of gelatinous material is perceived for all the investigated periods of time, which was already expected because of the hydrophilicity of the gelatin chains with water.

At the end of the $48 \mathrm{~h}$ of analysis, the results show that the lowest solubility and, consequently, the best matrix for use in biomedical devices is attributed to genipin, one of the proposed crosslinkers for the replacement of glutaraldehyde.

In contrast to this result, the EDC molecule appears to be the worst crosslinker according to the results of the analysis. Its percentages of dissolved gelatin were higher than the other crosslinkers at all times studied.

It can also be observed that, despite having relatively high percentages of dissolved gelatine (compared to the other crosslinkers) in the initial analysis times, fructose presents results close to that of glutaraldehyde and is even lower when the analysis is carried out after $48 \mathrm{~h}$. Considering that the possible application of this material will be for the controlled release of drug, the solubility profile of this sample gives a positive aspect to the microspheres cross-linked with fructose since it aims at a device that partially solubilizes in the initial moments (to release part of the pharmaceutical load ) and remain unsolvable for many hours.

\subsection{Gelatin microspheres with magnetic properties}

The magnetic material was added to the gelatin before the formation of crosslinked microspheres with fructose. We used two types of magnetic material (fluid or solid) and synthesized magnetite using ferrous chloride or ferrous sulfate. Table 3 shows the composition of samples obtained by saturation magnetization.

The most important magnetic property for medical application is the saturation magnetization. Systems for controlled release of drugs should have high saturation magnetization values because the magnetic gradient

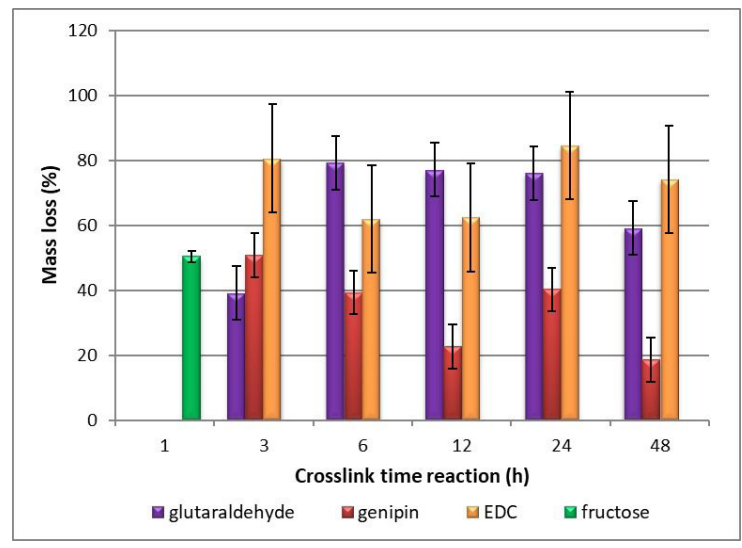

Figure 2. Enzymatic degradation of the samples.

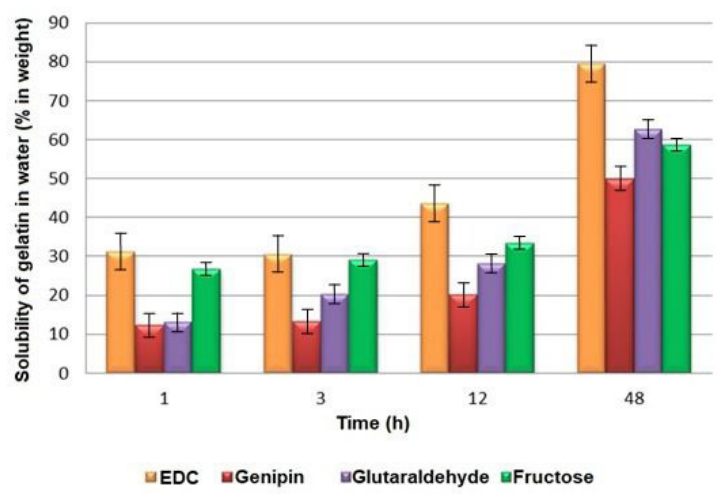

Figure 3. Water solubility of the samples.

Table 3. Magnetic properties of the magnetite and magnetic gelatin microspheres.

\begin{tabular}{cccc}
\hline Sample & Description & Ms (emu/g) & $\mathbf{H}_{\mathbf{C}}(\mathbf{G})$ \\
\hline Mag1 & magnetite obtained by ferrous sulfate & 53.90 & 37.82 \\
\hline Mag2 & magnetite obtained by ferrous chloride & 61.60 & 18.91 \\
\hline MEFS & $\begin{array}{c}\text { microspheres based on gelatin, fructose and } \\
\text { magnetite obtained by ferrous sulfate }\end{array}$ & 9.96 & 38.54 \\
\hline MEFC & $\begin{array}{c}\text { microspheres based on gelatin, fructose and } \\
\text { magnetite obtained by ferrous chloride }\end{array}$ & 29.6 & 20.67 \\
\hline MFFS & $\begin{array}{c}\text { magnetic fluid based on gelatin and magnetite } \\
\text { obtained by ferrous sulfate }\end{array}$ & 8.19 & 19.24 \\
\hline
\end{tabular}


decreases with increasing distance between the biomedical device and the external magnetic field. The target tissues for controlled-release are at a depth of 10 to $20 \mathrm{~cm}$ in the body, depending on the body mass of the patient and application site $^{34}$. Thus, microspheres with low saturation magnetization values require strong external magnetic fields to achieve the magnetic gradient ideal for transporting the drug to the desired location.

The magnetite obtained from ferrous chloride had saturation magnetization of $61.60 \mathrm{emu} / \mathrm{g}$ whereas that obtained with ferrous sulfate had 53.90 emu/g magnetization. Compared with values found in the literature ${ }^{33,35}$, the two solid magnetites have suitable magnetic susceptibility for the formulation of biomedical systems. This does not apply to magnetic fluids because of its low saturation magnetization (1.49 emu/g). The saturation magnetization of the magnetite obtained from ferrous chloride (MG2) was the highest among all magnetic materials obtained. This result indicates that the choice of counterion in the the ionic solid that provides the $\mathrm{Fe}^{+2}$ ion is essential to obtain the desired properties of the magnetite produced.

When magnetic gelatin microspheres were analyzed, the magnetization values were lower than can be predicted from the saturation magnetization of the pure ferromagnetic material as it has been reported in the literature ${ }^{36}$. However, we compared the magnetization values of the samples obtained in this study with the literature magnetization values. The MEFS and MFFS samples exhibited saturation magnetization values below those found in literature, which are of the order of $25 \mathrm{emu} / \mathrm{g}^{33}$ and $20 \mathrm{emu} / \mathrm{g}^{37}$. However, for the MEFC sample, a value higher than those described in the literature was found $(29,6 \mathrm{emu} / \mathrm{g})$. For use in a biological environment, another desirable property is the presence of superparamagnetism. In the absence of this property, the particles tend to agglomerate, which can lead to blocked blood vessels. The superparamagnetism of a sample can be confirmed by absence of hysteresis in the magnetization curve. Figure 4 shows the magnetization curve of sample $\mathrm{MEFC}$, indicating the sample has superparamagnetic behavior.

The SEM images (Figure 5) show that the MEFC magnetic gelatin microsphere have regular spherical morphology and size of about $50 \mu \mathrm{m}$. The increase in image magnification of the microsphere shows a smooth surface, for the most part, with presence of imperfections caused by collision with other microspheres present in the emulsion during preparation. The SEM-EDS analysis of iron distribution reveals that the microspheres incorporated magnetite uniformly distributed over their entire surface and did not form clusters.

\subsection{Doxorubicin loading and release}

Because of their solubility and appropriate magnetic properties, the fructose crosslinked gelatin microspheres containing magnetite obtained with ferrous chloride (MEFC) were subjected to doxorubicin loading and release tests. For comparison, microspheres prepared in the absence of magnetic material were also subjected to the same tests. The microspheres were immersed for different periods of time in doxorubicin $(0.5 \mathrm{mg} / \mathrm{mL}$ aqueous solution $)$. The drug concentrations in the microspheres at different times can be seen in Figure 6.

The microspheres prepared in the absence of magnetic material showed higher drug concentrations. These results can be explained by the way the drug binds to the gelatin chains, as can be seen in Figure 7. It is known that doxorubicin binds to gelatin using an acid labile hydrazone through glycylglycinic terminals originally present in proteins ${ }^{38}$. These terminals have amidic portions, and doxorubicin binds to the amino acid, replacing the two hydrogens of the amide terminals. When the microspheres have magnetic material added to them, the number of available glycylglycinic terminals decreases considerably because magnetite has strong electrostatic interaction with the $\mathrm{NH}_{2} / \mathrm{NH}_{3}{ }^{+}$groups present in the gelatin. For this reason, there is a greater possibility of forming new drug-gelatin linkages in microspheres without magnetite.

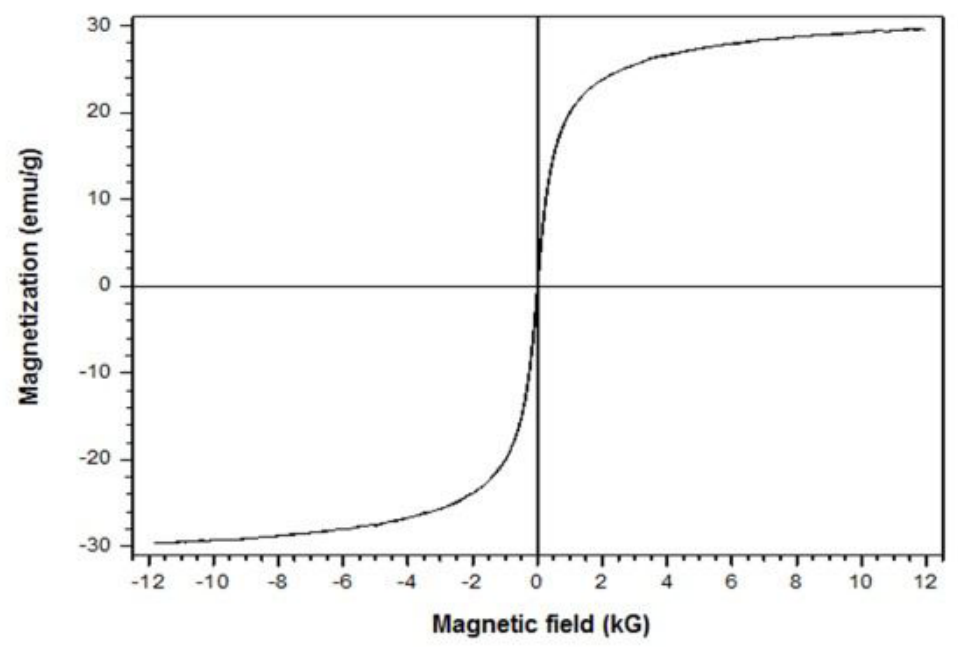

Figure 4. Variation of magnetization with the applied field of microspheres based on gelatin, fructose and magnetite obtained by ferrous sulfate (MEFC). 


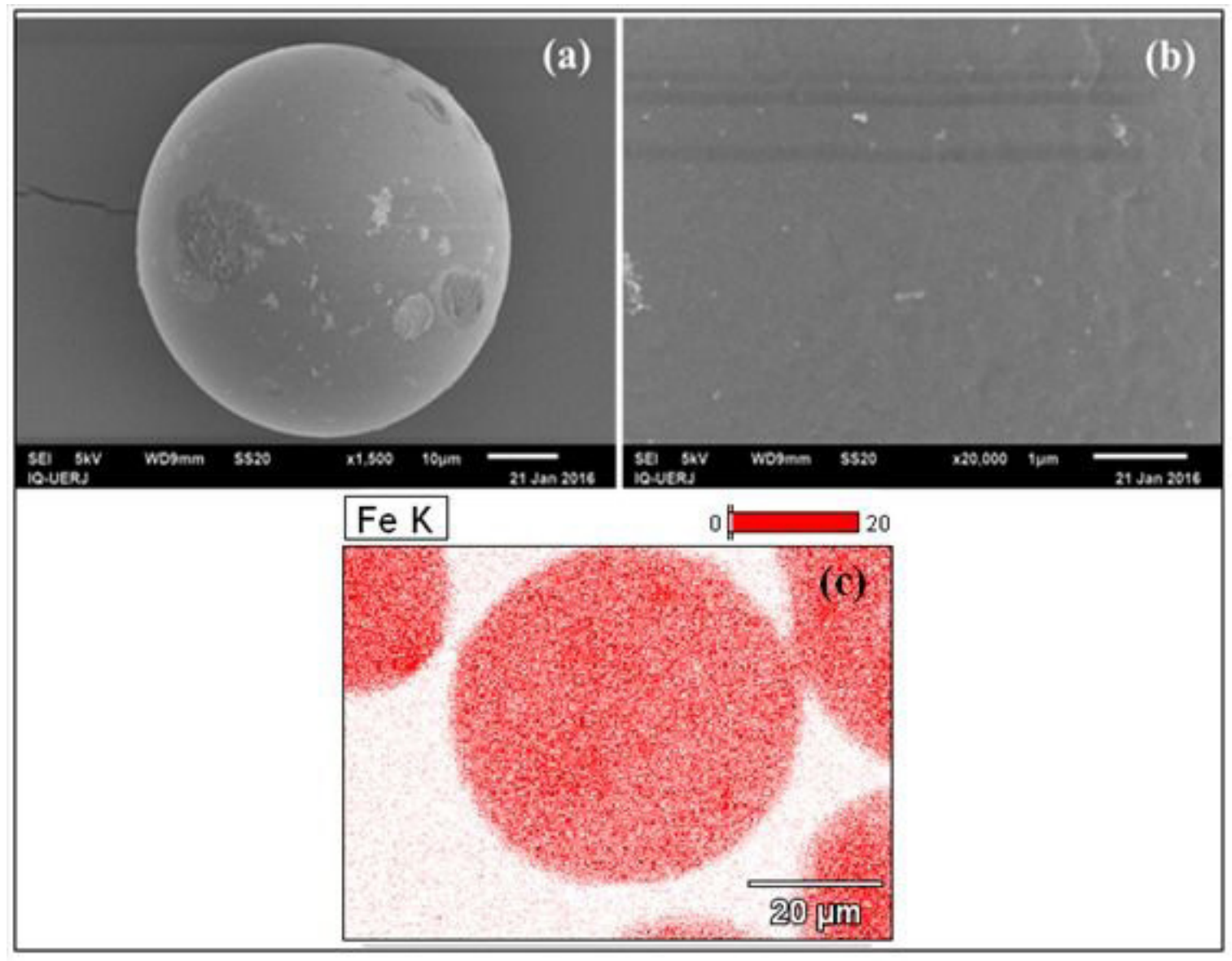

Figure 5. Scanning electron microscopy of microspheres based on gelatin, fructose and magnetite obtained by ferrous chloride (MEFC). (a) microsphere at $1500 \mathrm{X}$ (b) microsphere surface at 20000X and (c) energy dispersive X-ray spectroscopy of iron distribution.

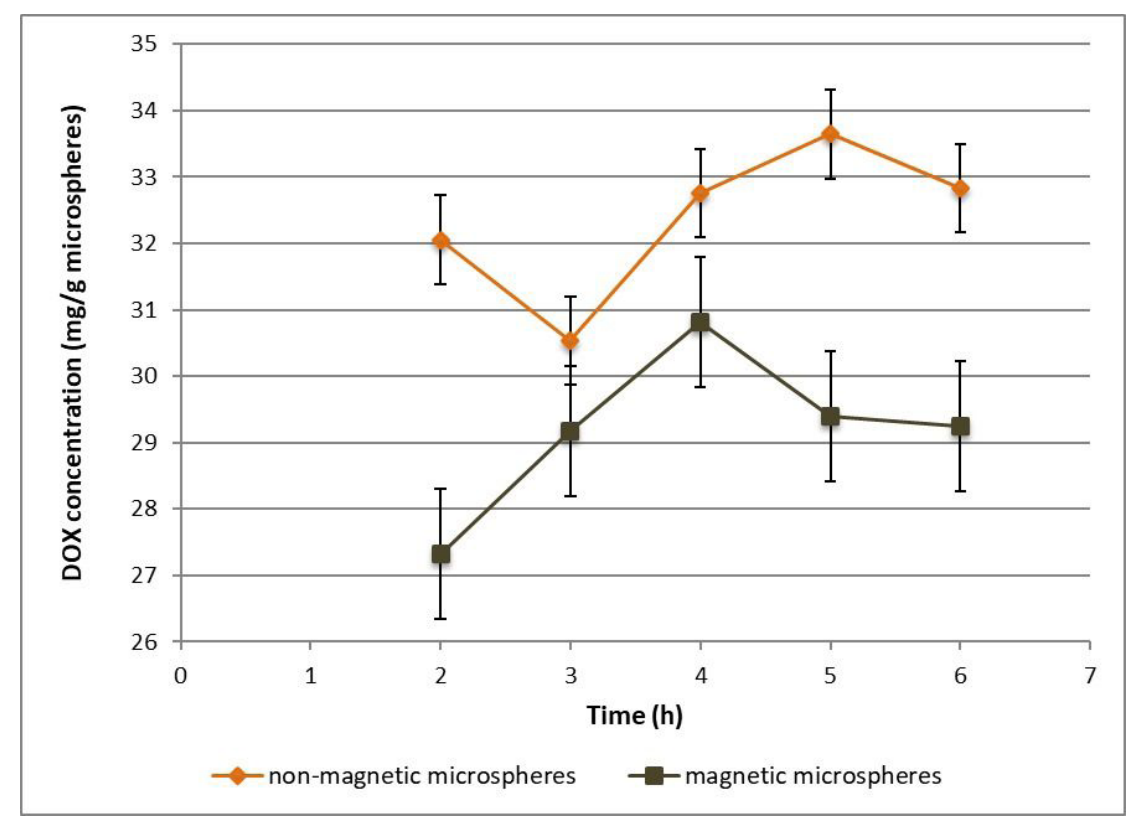

Figure 6. Doxorubicin loading on microspheres based on gelatin and fructose (non-magnetic microspheres) and microspheres based on gelatin, fructose magnetite obtained by ferrous chloride (magnetic microspheres). 
The microspheres were immersed for different periods of time in PBS. The samples were submitted to constant, oscillatory or none magnetic field. For comparison, nonmagnetic microspheres prepared were also subjected to the same tests. Figure 8 presents these release results. As seen at Figure 6, the doxorubicin incorporation by non-magnetic microspheres was greater than others samples. Because of this, their release was also greater for all analyzed time periods. This release profile does not have ideal characteristics to drug delivery systems because in these devices the goal is release the drug gradually.

When comparing drug release for magnetic and nonmagnetic systems, the release is significantly reduced by the presence of magnetite. When comparing the three magnetic systems, there is no statistical difference of release in the first six hours of contact with the aqueous medium. After that, there are different release profiles, which depend directly on the manner in which the microspheres are subjected to external magnetic field. Smaller drug release percentages were obtained for samples that were not subjected to any external field and the highest percentages were observed for those with the influence of constant magnetic field. The presence of external magnetic field causes agitation of the samples because there is always a magnetic moment alignment trend. This agitation becomes more noticeable when the magnetic field is oscillating and the particles are highly magnetic and display superparamagnetism. This agitation facilitates drug releaase compared with the release when the particles are not subjected to external magnetism. However, when the agitation is high and with a high-speed alignment/

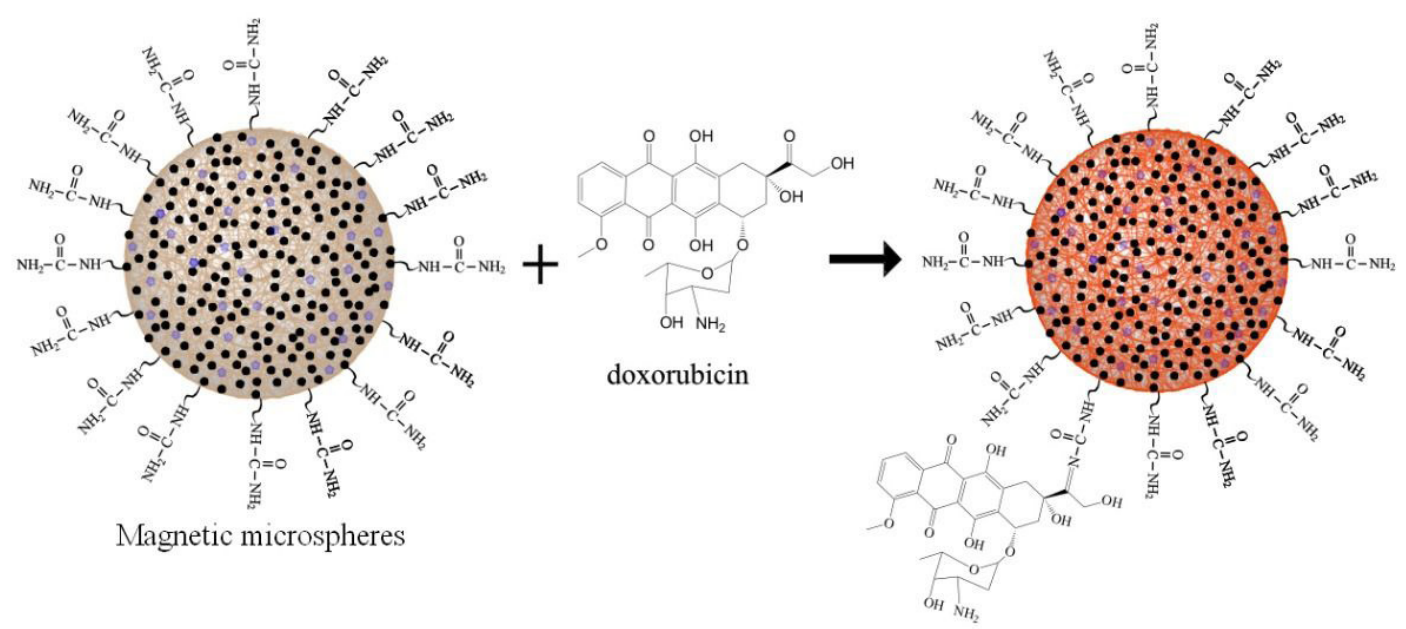

Figure 7. Scheme of doxorubicin bonding to gelatin glycylglycine terminals.

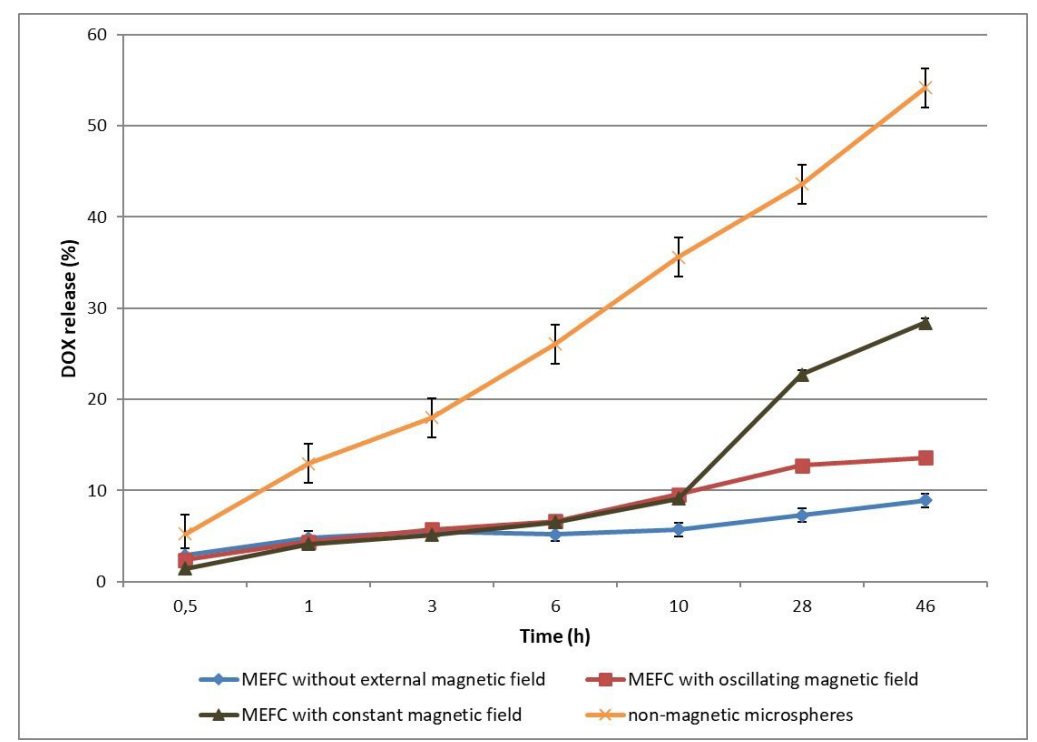

Figure 8. Microspheres based on gelatin, fructose magnetite obtained by ferrous chloride (sample MEFC) Doxorubicin release. 
dealignment of the microspheres' magnetic domains, there is significant interference with porosity. This interference usually causes closing of the pores and, consequently, difficulty in release of drug molecules. The obtainment of four different release profiles of doxorubicin was a positive result, because it enables tailoring the drug release to the pharmacological needs of each patient by only changing the gelatinous matrix or the way the patient is submitted to the external magnetic field.

\section{Conclusions}

A doxorubicin delivery system based on magnetic gelatin microspheres crosslinked by fructose was successfully developed. A superparamagnetic system was obtained with high saturation magnetization and low cytotoxiity of the crosslinker (fructose) and various release profiles. Among the crosslinkers studied, genipin was the most efficient to reduce the solubility of the gelatin microspheres in water. The magnetite properties were dependent on the chemical reagent used in the synthesis. When ferrous chloride was employed, the Ms showed the highest values, and its use in magnetic gelatin microspheres allowed the production of systems with high magnetization. Higher percentages of loading and drug release were obtained for non-magnetic microspheres. For magnetic microspheres, the application of external magnetic field (constant or oscillating) did not provoke a change in doxorubicin release profile until six hours. After that, a larger percentage of drug was released when constant external magnetic field was used. Furthermore, lower percentages of release were obtained without a magnetic field and intermediate percentages for oscillating magnetic field. This way, it was possible to produce systems for release of doxorubicin with four different profiles, allowing tailoring the release to the patient's needs.

\section{Acknowledgments}

The authors thank the financial support of the Carlos Chagas Filho Foundation for Research Support of the State of Rio de Janeiro (FAPERJ) and National Council for Scientific and Technological Development (CNPq), Proc.: 477907/2013-0.

\section{References}

1. Frachini ECG, Petri DFS. Magneto-responsive hydrogels: preparation, characterization, biotechnological and environmental applications. J Braz Chem Soc. 2019;30(10):2010-28.

2. Patil-Sen Y, Torino E, De Sarno F, Ponsiglione AM, Chhabria $\mathrm{V}$, Ahmed W, et al. Biocompatible superparamagnetic core-shell nanoparticles for potential use in hyperthermia-enabled drug release and as an enhanced contrast agent. Nanotechnology. 2020;31:375102. http://dx.doi.org/10.1088/1361-6528/ab91f6.

3. Al-Rawi NN, Anwer BA, Al-Rawi NH, Uthman AT, Ahmed IS. Magnetism in drug delivery: the marvels of iron oxides and substituted ferrites nanoparticles. Saudi Pharm J. 2020;28(7):87687.

4. Roullin VG, Deverre JR, Lemaire L, Hindré F, Venier-Julienne $\mathrm{MC}$, Vienet $\mathrm{R}$, et al. Anti-cancer drug diffusion within living rat brain tissue: an experimental study using $[3 \mathrm{H}]$ (6)-5-fluorouracil-loaded PLGA microspheres. Eur J Pharm Biopharm. 2002;53(3):293-9.
5. Saravanan M, Bhaskar K, Maharajan G, Pillai KS. Ultrasonically controlled release and targeted delivery of diclofenac sodium via gelatin magnetic microspheres. Int J Pharm. 2004;283(12):71-82.

6. Cukierman E, Khan DR. The benefits and challenges associated with the use of drug delivery systems in cancer therapy. Biochem Pharmacol. 2010;80(5):762-70.

7. Vasir JK, Tambwekar K, Garg S. Bioadhesive microspheres as a controlled drug delivery system. Int J Pharm. 2003;255(12):13-32.

8. Leo E, Vandelli MA, Cameroni R, Forni F. Doxorubicinloaded gelatin nanoparticles stabilized by glutaraldehyde: involvement of the drug in the cross-linking process. Int J Pharm. 1997;155(1):75-82.

9. Rathna GVN. Gelatin hydrogels: enhanced biocompatibility, drug release and cell viability. J Mater Sci Mater Med. 2008;19(6):2351-8.

10. Lee SJ, Yhee JY, Kim SH, Kwon IC, Kim K. Biocompatible gelatin nanoparticles for tumor-targeted delivery of polymerized siRNA in tumor-bearing mice. J Control Release. 2013;172(1):358-66.

11. Santoro M, Tatara AM, Mikos AG. Gelatin carriers for drug and cell delivery in tissue engineering. J Control Release. 2014;190:210-8.

12. Khan PA, Ismail SJ, Gani SR. Gelatin beads as sustained release drug delivery system. J Innov Pharm Biol Sci. 2014;1(1):10-6.

13. Sharma J, Bohidar HB. Gelatin-glutaraldehyde supramolecular structures studied by laser light scattering. Eur Polym J. 2000;36(7):1409-18.

14. Dinarvand R, Mahmoodi S, Farboud E, Salehi M, Atyabi F. Preparation of gelatin microspheres containing lactic acid--effect of cross-linking on drug release. Acta Pharm. 2005;55(1):57-67.

15. Madhusudana Rao K, Krishna Rao KSV, Ramanjaneyulu G, Ha CS. Curcumin encapsulated $\mathrm{pH}$ sensitive gelatin based interpenetrating polymeric network nanogels for anti cancer drug delivery. Int J Pharm. 2015;478(2):788-95.

16. Gaihre B, Khil MS, Lee DR, Kim HY. Gelatin-coated magnetic iron oxide nanoparticles as carrier system: drug loading and in vitro drug release study. Int J Pharm. 2009;365(1-2):180-9.

17. Khor E. Methods for the treatment of collagenous tissues for bioprostheses. Biomaterials. 1997;18(2):95-105.

18. Chattopadhyay S, Raines RT. Review collagen-based biomaterials for wound healing. Biopolymers. 2014;101(8):821-33.

19. Lien S-M, Li W-T, Huang T-J. Genipin-crosslinked gelatin scaffolds for articular cartilage tissue engineering with a novel crosslinking method. Mater Sci Eng C. 2008;28(1):36-43.

20. Cortesi R, Nastruzzi C, Davis SS. Sugar cross-linked gelatin for controlled release: microspheres and disks. Biomaterials. 1998;19(18):1641-9.

21. Schuler BJ. Evaluation of novel cross-linking agents for gelatin/ collagen matrices [dissertation]. Morgantown, West Virginia: West Virginia University; 2004. http://dx.doi.org/10.33915/etd.2092.

22. Souza J, Silva M, Costa M. Potential doxorubicin delivery system based on magnetic gelatin microspheres crosslinked with sugars. Polímeros. 2018;28(2):131-8.

23. Adhirajan N, Shanmugasundaram N, Babu M. Gelatin microspheres cross-linked with EDC as a drug delivery system for doxycyline: development and characterization. J Microencapsul. 2007;24(7):647-59.

24. Siimon K, Siimon H, Järvekülg M. Mechanical characterization of electrospun gelatin scaffolds cross-linked by glucose. J Mater Sci Mater Med. 2015;26(1):5375.

25. Uranga J, Leceta I, Etxabide A, Guerrero P, De La Caba K. Cross-linking of fish gelatins to develop sustainable films with enhanced properties. Eur Polym J. 2016;78:82-90.

26. Widder KJ, Senyei AE, Ranney DF. Magnetically responsive microspheres and other carriers for the biophysical targeting of antitumor agents. Adv Pharmacol Chemother. 1979;16:213-71. 
27. Mody VV, Cox A, Shah S, Singh A, Bevins W, Parihar H. Magnetic nanoparticle drug delivery systems for targeting tumor. Appl Nanosci. 2014;4(4):385-92.

28. Sadighian S, Rostamizadeh K, Hosseini-Monfared H, Hamidi M. Doxorubicin-conjugated core-shell magnetite nanoparticles as dual-targeting carriers for anticancer drug delivery. Colloids Surf B Biointerfaces. 2014;117:406-13.

29. Coukell AJJJ, Spencer CMMM. Polyethylene glycol-liposomal doxorubicin. A review of its pharmacodynamic and pharmacokinetic properties, and therapeutic efficacy in the management of AIDS-related Kaposi's sarcoma. Drugs. 1997;53(3):520-38.

30. Fan H, Dash AK. Effect of cross-linking on the in vitro release kinetics of doxorubicin from gelatin implants. Int J Pharm. 2001;213(1-2):103-16.

31. Hong RY, Pan TT, Han YP, Li HZ, Ding J, Han S. Magnetic field synthesis of Fe3O4 nanoparticles used as a precursor of ferrofluids. J Magn Magn Mater. 2007;310(1):37-47.

32. Vandelli MA, Rivasi F, Guerra P, Forni F, Arletti R. Gelatin microspheres crosslinked with D, L-glyceraldehyde as a potential drug delivery system: preparation, characterisation, in vitro and in vivo studies. Int J Pharm. 2001;215(1-2):175-84.
33. Choubey J, Bajpai AK. Investigation on magnetically controlled delivery of doxorubicin from superparamagnetic nanocarriers of gelatin crosslinked with genipin. J Mater Sci Mater Med. 2010;21(5):1573-86.

34. Arruebo M, Fernández-Pacheco R, Ibarra MRR, Santamaría J. Magnetic nanoparticles for drug delivery. Nano Today. 2007;2(3):22-32.

35. Huang LY, Yang MC. Behaviors of controlled drug release of magnetic-gelatin hydrogel coated stainless steel for drugeluting-stents application. J Magn Magn Mater. 2007;310(2, Pt 3):2874-6.

36. Philippova O, Barabanova A, Molchanov V, Khokhlov A. Magnetic polymer beads: recent trends and developments in synthetic design and applications. Eur Polym J. 2011;47(4):54259.

37. Yilmaz H, Sanlier SH. Preparation of magnetic gelatin nanoparticles and investigating the possible use as chemotherapeutic agent. Artif Cells Nanomed Biotechnol. 2013;41(2):69-77.

38. Wu DC, Cammarata CR, Park HJ, Rhodes BT, Ofner CM 3rd. Preparation, drug release, and cell growth inhibition of a gelatin: doxorubicin conjugate. Pharm Res. 2013;30(8):2087-96. 